\title{
A FIXED POINT THEOREM FOR $(n-2)$-CONNECTED n-POLYHEDRA
}

\author{
ROGER WAGGONER
}

\begin{abstract}
The main result of this paper is that, for $n \geqq 4$, a finite $(n-2)$-connected polyhedron $K$ of dimension $n$ admits a fixed point free map if either $H_{n-1}(K ; Q)$ or $H_{n}(K ; Q)$ is nonzero, where $Q$ is the field of rational numbers. This result is obtained by first retracting $K$ onto a subpolyhedron $C$ of dimension $n$ or $n-1$ which has no local separating points. It is then shown that $C$ admits a map with Lefschetz number zero, and it follows from a theorem of Shi that $C$ does not have the fixed point property. The proof involved may also be applied when $K$ is a 3-dimensional simply connected polyhedron and the subpolyhedron $C$ is also of dimension 3 .
\end{abstract}

1. Introduction. The main purpose of this paper is to show that, for $n \geqq 4$, a finite $(n-2)$-connected polyhedron $K$ of dimension $n$ admits a fixed point free map if either $H_{n-1}(K ; Q)$ or $H_{n}(K ; Q)$ is nonzero. This result may be regarded as a higher dimensional analogue of the wellknown fact that if $H_{1}(K ; Q) \neq 0$, then the space $K$ admits a fixed point free map (obtained by retracting $K$ onto a simple closed curve). The proof of the present theorem is obtained by retracting $K$ onto a subpolyhedron $C$ of dimension $n$ or $n-1$ which has no local separating points, and then constructing a map of $C$ which has Lefschetz number 0 . It follows from a theorem of Shi [3] that $C$ fails to have the fixed point property. The reader may use Fadell [1] as a general reference.

A map is a continuous function. If $f: X \rightarrow Y$ is a map, then $M(f)$ will denote the mapping cylinder of $f$. Unless otherwise indicated, $H(X)$ will denote the singular homology of $X$ with integer coefficients, while $\tilde{H}(X ; Q)$ denotes the reduced singular homology of $X$ with rational coefficients. If $c$ is a cocycle of $X$, then [c] will be the image of $c$ in the cohomology group of $X$. A bouquet of $m$-spheres is a CW complex consisting of a base point $x_{0}$ and a collection of $m$-cells $\left\{S_{\alpha}\right\}$ whose boundaries are attached to $x_{0}$. A polyhedron $K$ satisfies the Shi condition if the dimension of $K$ is at least 3 and $K$ has no local separating points. The $j$-sphere is $S^{j}$. The term "fixed point property" will be abbreviated "f.p.p.".

Presented to the Society, January 20,1972; received by the editors May 3, 1971 and, in revised form, July 7, 1971.

AMS 1969 subject classifications. Primary 5485, 5460.

Key words and phrases. Local separating point, mapping cylinder, retraction, fixed point property.

(c) American Mathematical Society 1972 
2. The Theorem. The following lemma gives a sufficient condition for retracting a $\mathrm{CW}$ complex of dimension $m+1 \geqq 3$ onto an $m$-dimensional subcomplex which is a bouquet of spheres.

Lemma. Let $K$ be a $\mathrm{CW}$ complex of dimension $m+1 \geqq 3$, let $B$ be an $m$-dimensional bouquet of spheres which is a subcomplex of $K$, and let $i$ : $B \subset K$. If $i_{*}: H_{m}(B) \rightarrow H_{m}(K)$ has a left inverse $h$, then $B$ is a retract of $K$.

Proof. Cellular homology is used for this proof, and the obstruction theory involved is found in $\mathrm{Hu}$ [2]. Consider the following commutative diagram.

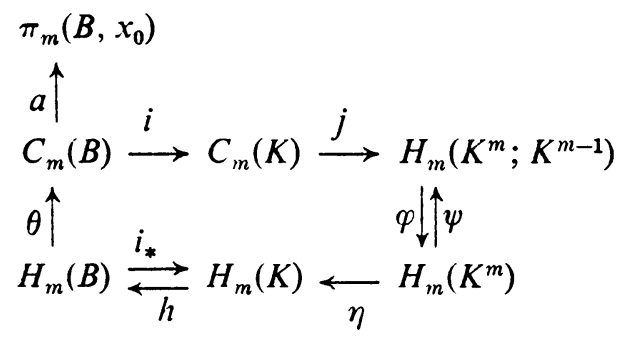

$C_{m}(B)$ and $C_{m}(K)$ are groups of cellular chains, $a$ is a cocycle in $Z^{m}\left(B ; \pi_{m}\left(B, x_{0}\right)\right), K^{m}$ and $K^{m-1}$ are skeleta of $K, \theta$ and $j$ are isomorphisms, $\eta$ and $\psi$ are induced by inclusion, and $\phi \psi$ is the identity. Then $a \theta h \eta \varphi j$ is an $m$ cocycle for $K$. Now $i^{*}[a \theta h \eta q j]=[a \theta h \eta \varphi j i]=[a]$, so $i^{*}: H^{m}\left(K ; \pi_{m}\left(B, x_{0}\right)\right) \rightarrow$ $H^{m}\left(B ; \pi_{m}\left(B, x_{0}\right)\right)$ is an epimorphism. Thus $\delta: H^{m}\left(B ; \pi_{m}\left(B, x_{0}\right)\right) \rightarrow$ $H^{m+1}\left(K, B ; \pi_{m}\left(B, x_{0}\right)\right)$ is zero. But the image of $\delta$ contains the obstruction to extending a map $B \rightarrow B$ to a map $K \rightarrow B$. Since any such map may be extended, $B$ is a retract of $K$.

THEOREM. If $K$ is a finite (n-2)-connected polyhedron of dimension $n \geqq 4$ and $\tilde{H}(K: Q) \neq 0$, then $K$ does not hate the f.p.p.

Proof. Let us first suppose that $K$ satisfies the Shi condition. For either $j=n$ or $j=n-1, H_{j}(K ; Q) \neq 0$. Since the Hurewicz homomorphism $\pi_{j}(K) \rightarrow H_{j}(K)$ is onto, there is a cellular map $f: S^{j} \rightarrow K$ such that $f_{*}$ : $H_{j}\left(S^{j}\right) \rightarrow H_{j}(K)$ is a monomorphism and the image of $f_{*}$ is a direct factor of $H_{j}(K)$. This implies that $f_{*}$ has a left inverse. Thus if $i: S^{j} \subset M(f), i_{*}$ also has a left inverse. Applying the lemma, $S^{j}$ is a retract of $M(f)$ and hence $M(f)$ admits a fixed point free map. Thus $K$ admits a map whose Lefschetz number is 0 , and from results in [3] it follows that $K$ has a fixed point free map.

For the general case, let $r_{1}, \cdots, v_{q}$ be the vertices of $K$, and let $C_{1}, \cdots$, $C_{p}$ be the closures of the components of $K\left\{v_{1}, \cdots, v_{q}\right\}$. Then there is an 
$(n-2)$-connected $C_{k}$ which satisfies the Shi condition, for which $\hat{H}\left(C_{k} ; Q\right) \neq$ 0 . Since $C_{k}$ is a retract of $K$ which admits a fixed point free map, $K$ does also.

REMARK. The proof of the theorem may also be applied if the dimension of $K$ is 3 and there is some $C_{k}$ of dimension 3 with $\tilde{H}\left(C_{k} ; Q\right) \neq 0$. For example, if the dimension of $K$ is $3, K$ is simply connected, and $H_{3}(K ; Q) \neq 0$, then $K$ fails to have the f.p.p.

\section{BIBLIOGRAPHY}

1. E. Fadell, Recent results in the fixed point theory of continuous maps, Bull. Amer. Math. Soc. 76 (1970), 10-29.

2. S. T. Hu, Homctopy theory, Pure and Appl. Math., vol. 8, Academic Press, New York, 1959. MR 21 \#5186.

3. Shih Ken-hua, On least number of fixed points and Nielsen numbers, Acta Math. Sinica 16 (1966), 223-232=Chinese Math.-Acta 8 (1966), 234-243. MR 35 \#1004.

Department of Mathematics, University of Southwestern Louisiana, LafaYETTE, LOUISIANA 70501 